\title{
Intra-Operative and Anatomic Verification of a TKA Computational Model for Pre-Operative Surgical Planning
}

\author{
Joshua G Twiggs ${ }^{1}$, Edgar A. Wakelin ${ }^{1}$, Justin Roe ${ }^{2}$, Brett Fritsch ${ }^{3}$, Brad \\ Miles $^{1}$
}

1360 Knee Systems, Sydney, Australia

${ }^{2}$ North Sydney Orthopaedic and Sports Medicine Clinic, Sydney, Australia

${ }^{3}$ Sydney Orthopaedic Research Institute Sydney, Australia

joshua@kneesystems.com, brad@_kneesystems.com

\begin{abstract}
A promising measure of dynamic outcomes in Total Knee Arthroplasty (TKA) is the simulation of joint dynamics. These simulations are potentially useful for pre-operative planning, but are not yet validated for patient-specific variations in anatomy, which forms the aim of this paper. 284 patients from a database of total knee arthroplasty patients were analysed using a pre-operatively defined simulation predicting post-operative knee dynamics; each patient had previously undergone pre- and post-operative CT imaging and had been assessed using the Knee Injury and Osteoarthritis Outcome (KOOS) score at 6 months following surgery. A significant correlation was found between the simulated contact force and laxity in mid-flexion $(r=-0.452, p<0.0001)$, and between the mediolateral difference in contact force and difference in distal-vs-posterior femoral collateral ligament offset $(\mathrm{r}=-0.473, \mathrm{p}<0.0001)$. A significant difference of $5 \mathrm{KOOS}$ pain points $(\mathrm{p}=0.02)$ was found for patients with unusually low or high simulated contact force compared to normal. These results indicate the preoperative simulation is capable of distinguishing patient-specific kinematics prior to surgery, thereby demonstrating the utility of this simulation for making pre-surgical predictions of patient-specific kinematics and patient-reported outcomes.
\end{abstract}




\section{Introduction}

Component position and alignment in Total Knee Arthroplasty (TKA) are known to impact the kinematics of the knee [1, 2], which in turn have been shown to affect patient outcome [3]. TKA dynamic outcomes can be measured in a variety of ways, such as gait analysis, video fluoroscopy [20] and mechanical rig testing and have been used in design validation studies [27]. Such techniques however are unsuitable or not scalable for routine pre-operative planning. Dynamic knee computer simulations are a promising scalable alternative and allow the impact of both patient and surgical factors on joint dynamics and patient outcome to be studied prior to surgery [4]. Although computation simulations are routinely validated through cadaveric studies under controlled conditions, intraoperative validation of simulated joint dynamics and how this relates to patient specific anatomy has not been achieved. Here we verify simulated joint dynamics generated by a computational knee model for routine pre-operative planning by comparison to data captured through a navigation platform.

\section{Method}

A database of TKA Patients undergoing surgery from 1-Jan-2014 operated on by 9 surgeons, who received a pre-operative and post-operative $\mathrm{CT}$ and 6-month post-operative Knee Injury and Osteoarthritis Outcome (KOOS) score were assessed. All knees received either a CR or PS OMNI (Raynham, MA) APEX prosthesis and a dome patella button. A subset of these patients had surgery performed with the OMNIbotics navigation system from which the width of the laxity profile in midflexion $\left(40^{\circ}\right)$ was extracted. Segmented pre-operative bones, patient specific landmarks and component geometries were registered to the post-operative $\mathrm{CT}$ to determine the achieved component placement as per the AURORA protocol [5].

A musculoskeletal computational model with similar boundary conditions to the Oxford Knee Rig developed and validated as described by Theodore et al. [6] was used to simulate post-TKA knee dynamics using Adams MSC software (Newport, CA), see Figure 1. During model generation, anatomic landmarks were recorded to generate patient specific bony axes and define soft-tissue attachment sites. The simulated tibiofemoral contact force and force difference between the medial and lateral condyles in mid-flexion was then compared to the mid-flexion laxity range as measured by the navigation system, the difference in distal to posterior femoral collateral ligament offset, and post-operative KOOS outcome scores.

\section{Results}

A total of 284 patients were identified in the database. A subset of 69 patients also had corresponding navigation data recorded during surgery. 59.5\% (169) were female and the average age was $68.9 \pm 11.6$ years.

A significant moderate-strong correlation was found between the simulated contact force in midflexion and navigated mid-flexion laxity $(\mathrm{r}=-0.452, \mathrm{p}<0.0001)$, see Figure $2 \mathrm{~A}$, indicating that the simulation is able to distinguish kinematics observed intra-operatively. The medio-lateral contact force difference also has a moderate-strong correlation with the difference in distal to posterior femoral collateral ligament offset $(r=-0.473, p<0.0001)$, see Figure 2B, indicating the difference in simulated contact force is driven by the anatomic position of the ligament insertion sites. 
The contact force in mid-flexion was then compared to the 6-month KOOS pain outcome scores. Patients were dichotomised into a medium range contact force between 1500-3000 simulated $\mathrm{N}$ and those outside this range, see Figure 2C. A significant difference of 5 KOOS points $(p=0.02)$ was found between these two groups, in which those within the range reported improved outcome.

\section{Discussion}

This study has shown statistically significant correlations between patient specific anatomy, simulated kinematics, and intra-operatively observed navigation data, indicating the pre-operative simulation is capable of distinguishing patient specific kinematics prior to surgery. Furthermore, the simulated forces are shown to significantly correlate with patient reported outcomes, confirming the clinical relevance of such simulations for identifying kinematics which may result in improved patient outcomes. Other biomechanical simulations which focus on mechanical design optimisation [7] require either invasive data capture or simulation times which are not scalable. As such this work represents the first verification of simulated kinematics for a dynamic pre-operative planning tool. Although soft tissue attachment sites are patient specific, future work will include methods to obtain patient specific soft tissue properties in a non-invasive way that can be achieved as part of routine pre-operative planning.

The validity of such tools will allow surgeons to plan and optimise surgical procedures on a patient specific basis. Using this tool, outlier kinematics which may not be easily identified from the anatomy or intra-operatively can be measured and analysed before entering the operating theatre. Component positioning may then be modified to generate knee kinematics that correlate with improved outcomes based on the implant of choice and the patient's own anatomy. By achieving optimised patient specific kinematics the persistently high dissatisfaction rate in TKA may be reduced.

\section{Conclusions}

This study shows statistically significant correlations between anatomic measurements, simulated kinematics and intra-operative navigation data. Verifying that the simulation can predict patient specific kinematics and provide recommendations for optimal patient specific TKA component placement.

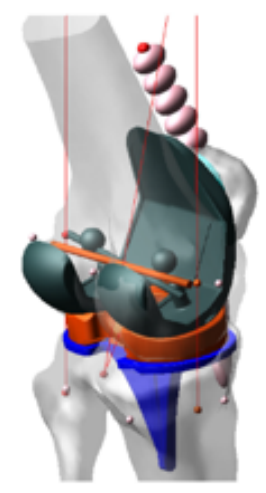

Figure 1: Computational knee model defined for routine pre-operative planning. 

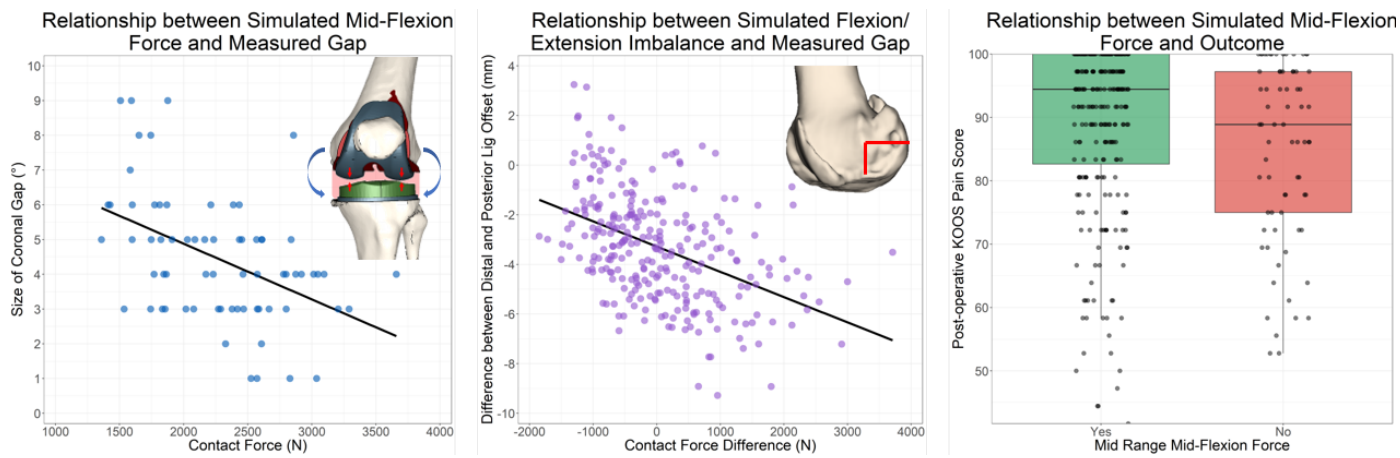

Figure 2: Comparison of simulated: A) tibio-femoral contact force and coronal gap size in mid-flexion. B) difference in tibio-femoral contact force and femoral ligament offset distance. C) Comparison of 6 month KOOS pain outcome and mean \pm SD simulated tibio-femoral contact force.

\section{References}

1. Harman, M.K., et al., Prosthesis alignment affects axial rotation motion after total knee replacement: a prospective in vivo study combining computed tomography and fluoroscopic evaluations. BMC musculoskeletal disorders, 2012. 13(1): p. 206.

2. Werner, F.W., et al., The effect of valgus/varus malalignment on load distribution in total knee replacements. Journal of biomechanics, 2005. 38(2): p. 349-355.

3. Huang, N.F., et al., Coronal alignment correlates with outcome after total knee arthroplasty: five-year follow-up of a randomized controlled trial. The Journal of arthroplasty, 2012. 27(9): p. 1737-1741.

4. Twiggs, J.G., et al., Patient-Specific Simulated Dynamics After Total Knee Arthroplasty Correlate With Patient-Reported Outcomes. The Journal of arthroplasty, 2018. 33(9): p. 2843-2850.

5. Wakelin, E.A., et al., Accurate determination of post-operative $3 D$ component positioning in total knee arthroplasty: the AURORA protocol. Journal of orthopaedic surgery and research, 2018. 13(1): p. 275.

6. Theodore, W., et al., Variability in static alignment and kinematics for kinematically aligned TKA. The Knee, 2017.

7. Rullkoetter, P.J., C.K. Fitzpatrick, and C.W. Clary, How Can We Use Computational Modeling to Improve Total Knee Arthroplasty? Modeling Stability and Mobility in the Implanted Knee. J Am Acad Orthop Surg, 2017. 25 Suppl 1: p. S33-S39. 